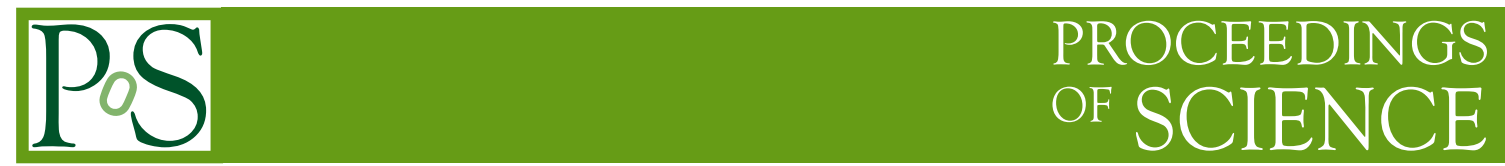

\title{
Wide-field VLBI Techniques: A Beginner's Guide
}

\section{John Morgan*}

ICRAR, Curtin University, Perth

E-mail: john.morganeicrar.org

Thanks to software correlators such as DiFX, the technique of wide-field VLBI has progressed dramatically in the last few years. In these proceedings I will try to summarise the current state of the art, provide some pointers for those who wish to do their own wide-field VLBI and suggest avenues for future work.

10th European VLBI Network Symposium and EVN Users Meeting: VLBI and the new generation of radio arrays

September 20-24, 2010

Manchester $U K$

*Speaker. 


\section{Introduction}

In standard VLBI only a very small fraction of the area of sky that the interferometer elements are sensitive to is imaged. In order to image flux very far from the phase centre, the correlated data must have very high time and spectral resolution to avoid averaging losses. Generating these large datasets (correlation) and reducing them (calibration and imaging) are the hurdles which must be overcome in order to fully exploit the inherent wide-field potential of any VLBI observation.

This potential for imaging large swathes of the primary beam was first explored about a decade ago as the PCs used for post-processing grew in power to the stage that imaging to the limits of typical correlator output could be attempted. This early work is summarised in Garrett et al. (1999). Pioneering work continued with Garrett et al. (2001), Garrett et al. (2005) and Lenc et al. (2008) pushing hardware correlators such as the JIVE Mark 4 to the limits of their spectral and time resolution (see Campbell 2004, for typical constraints on these parameters).

These projects demonstrated the usefulness of the wide-field VLBI technique, however all of these projects were hampered by three problems. Firstly, hardware correlators were incapable of correlating with sufficient resolution to image out far enough away from the phase centre that the entire primary beam could be imaged. This meant that very severe tapering of the UV coverage was required for the edge of the primary beam. Secondly, it was clear that though data-reduction software such as AIPS was capable of processing such large datasets, the performance was rather poor (Lenc et al. 2008). Finally, Lenc et al. (2008) and Middelberg et al. (2008) noted that astrometric accuracy was lost in the UV shifting procedure.

All of these problems have been addressed in recent years by developments in correlation and closer examination of the UV shifting technique. These advances are summarised in sections 2 and 3. Calibration techniques specific to wide-field VLBI are discussed in section 4. Possible avenues for future work are discussed in section 5 .

\section{Correlation}

As mentioned above, until recently wide-field imaging was limited principally by the maximum time and spectral resolution that hardware correlators are capable of producing. Software correlators generally perform much better in this regard, the only penalty being an increase in CPU time required to reach almost arbitrary resolution ${ }^{1}$. In particular DiFX (Deller et al. 2007) is easily configurable to have the necessary time and frequency resolution to image the entire primary beam even for low-frequency global VLBI with $25 \mathrm{~m}$ dishes.

\section{UV shifting}

Data reduction software such as AIPS is geared towards processing smaller UV datasets with adequate time and frequency resolution for imaging only a few million resolution units. For VLBI this corresponds to a tiny fraction of the primary beam. Some progress can be expected on this

\footnotetext{
${ }_{1}$ in principal this is possible for hardware correlators too, however without substantial programming to allow recirculation, the baseband data will have to be read multiple times. In addition, software correlators are much more easily upgradeable to provide further processing power since they are built on standard computer clusters
} 


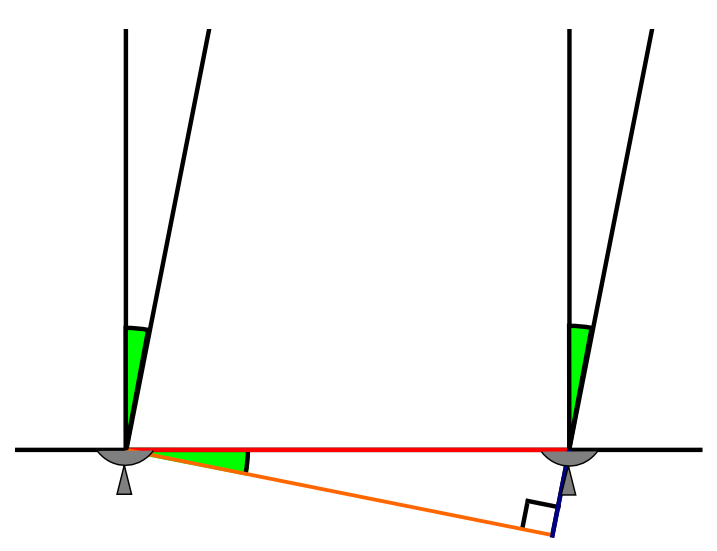

Figure 1: The geometry of a UV shift: moving to a new phase centre implies a different baseline vector and a delay differential requiring a non-dispersive phase shift.

front through the parallelisation of the imaging process (Bhatnagar 2009) however complications such as non-coplanar effects are likely to mean that making very large images will likely always come with a penalty.

Crucial to the success of wide-field VLBI is the concept of UV shifting. This allows multiple small datasets to be generated from a single very large UV dataset by shifting the phase centre of the dataset and averaging in time and frequency. UV shifting a visibility dataset consists of the following: the coordinates for the phase centre are changed; new baseline vectors are calculated and a phase shift is applied to each visibility to take account of the delay associated with the offset from the original phase centre (see fig. 1). The dataset can now be averaged

UV shifting can be done within AIPS ${ }^{2}$ however there are two problems. Firstly AIPS is not designed to handle extremely large datasets. Lenc et al. (2008) estimated that 6 years of computing time would be required to image the entire VLBA primary beam at $320 \mathrm{MHz}$. Wucknitz (These proceedings) has devised an iterative scheme to reduce this dramatically. However handling very large UV datasets is very unwieldy and may not be possible at all in AIPS for the largest datasets ${ }^{3}$.

\subsection{Accurate UV shifting}

The baseline vectors $u, v$ are used at the imaging stage to calculate the residual delay associated with an offset $(l, m)$ from the phase centre. They can be considered to be $\frac{\partial \tau}{\partial l}$ and $\frac{\partial \tau}{\partial m}$ respectively where $\tau$ is the residual delay across the baseline (zero at the phase centre). They can therefore be used to calculate the phase shift required to transform each visibility to the new phase centre. There are two problems with this approach which are discussed in detail in Morgan et al. (2010).

The delay is known to vary in a non-linear way across the wide field (see fig 2). This means that any scheme which assumes a linear change in delay with respect to $l$ and $m$ will inevitably lead to positional errors. These error cannot be calibrated out since this is inherently something

\footnotetext{
${ }^{2}$ either using the task UVFIX or at the time of imaging using the RASHIFT and DECSHIFT parameters for IMAGR

${ }^{3}$ Specifically there is a limitation on $n_{I F} \times n_{\text {channels }} \times n_{\text {polarisations }}$ (currently 32768) when loading the dataset (See parameters MAXCIF and LMXCIF in FITLD.FOR and PUVD.INC).
} 


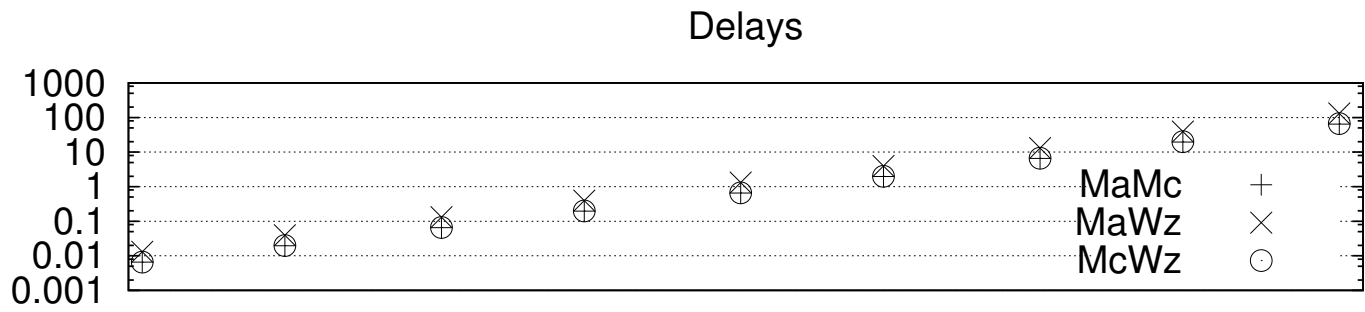

linear fit subtracted

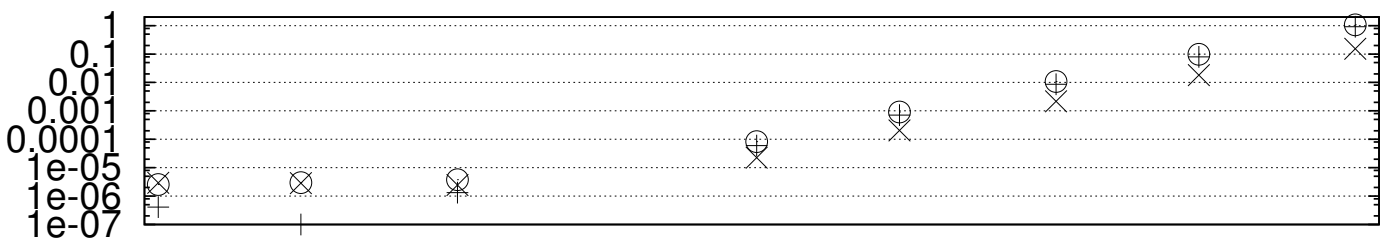

fractional error of linear fit

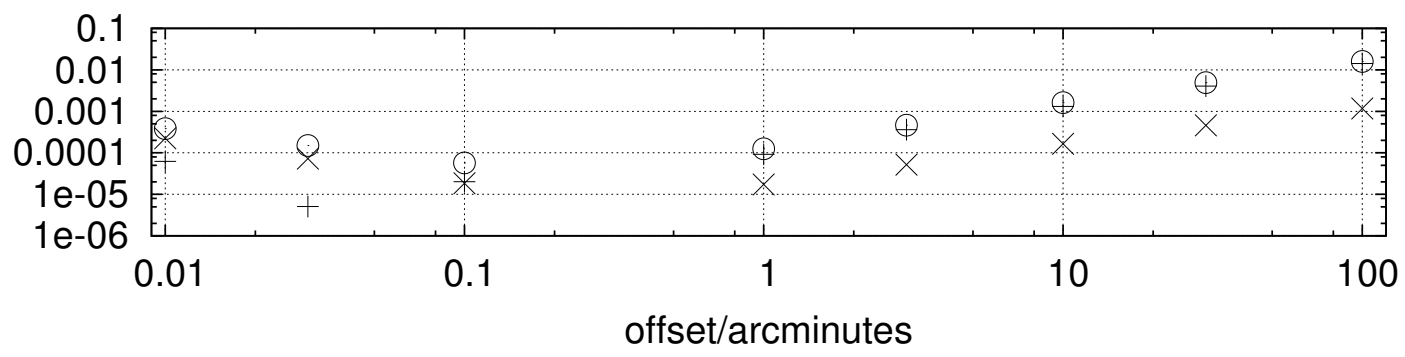

Figure 2: The residual delay as calculated with the correlator delay model (CALC 9) (in microseconds) with increasing offset.

which is applied to the target and not the calibrator source ${ }^{4}$. A far better scheme is to generate an entirely new delay model for the new phase centre (along with new baseline vectors) from scratch. The difference in delay between the original model and new one can be used to UV shift the data accurately and should produce results consistent with recorrelation with a new phase phase centre. Morgan et al. (2010) show that with a further slight refinement position errors across the shift are no more that one part in $10^{8}$ - an undetectable error for any conceivable shift for ground-based VLBI with parabolic antennas.

\subsection{Implementations}

This shifting software has been implemented in DiFX at the FITS conversion stage ${ }^{5}$. It has also been implemented with full accuracy within the DiFX software correlator itself (Deller et al. 2010). The data are correlated with extremely high time and frequency resolution, however this cumbersome dataset never leaves the computer memory: it is shifted multiple times and the enduser is supplied with an (almost) arbitrary number of small manageable datasets, each centred on a different area of interest.

\footnotetext{
${ }^{4}$ Practical steps can be taken to keep these errors negligable for astrometric observations, for example ensuring that the same phase centre is used at all epochs

${ }^{5}$ Non-standard software, available on request
} 


\section{Calibration}

Let us assume that the astronomer has a large number of standard VLBI datasets with phase centres scattered across the primary beam. To a first approximation, all flagging and calibration can be applied to all datasets equally. With the aid of pipelining tools such as ParselTongue (Kettenis et al. 2006) it is trivial to extend the calibration of a single dataset to all the others, and to image all datasets.

\subsection{Primary beam correction}

One refinement which can be made is to properly account for the effect of the primary beam attenuation. This is discussed in more detail in Morgan (these proceedings), Morgan et al. (2010) and Middelberg et al. (2010) and briefly summarised here. Essentially the amplitude of each visibility must be increased (and the weight appropriately decreased) to account for the primary beam attennuation. Morgan et al. (2010) approached the problem with a very simple scheme, modelling each antenna primary beam as a sinc function truncated at the first null. Middelberg et al. (2010) devised a more sophisticated scheme which took account of the non-radial and therefore time-varying (since the antennas have an alt-azimuth mount) primary beam due to to the feed offset (which is different for each polarisation).

When only the central part of the primary beam is of interest this correction is not critical. However for multiple pointings, particularly with heterogeneous arrays, the contribution of sensitivity from pointings where the target source is beyond the HPBW becomes important. In order for these data to be used the primary beams of the larger telescopes must be well characterised.

\subsection{Multiple weak-source self calibration}

Garrett et al. (2004) discussed the possibility of using multiple sources across the primary beam to allow self calibration even where no individual source is bright enough to self calibrate on alone. Now that calibrating with multiple phase centres is so easy, this technique comes into its own. This will open up many sources without a nearby calibrator to astrometric studies and improved calibration at L-band.

\section{Future Work}

Recent progress in the field of wide-field VLBI has made proposing, observing, correlating and analysing wide-field observations little more difficult that standard VLBI.

As discussed in Morgan et al. (2010) and Wucknitz (these proceedings), imaging the entire primary beam is possible even today. In the future, imaging the entire primary beam could become standard for L-band VLBI observations. As well as improving calibration for the primary target source, archived data of all detected sources would be very valuable for slow transient searches and population studies of compact objects, and would provide a useful complement to data taken with wide-field instruments at other resolutions and wavelengths. 


\section{Acknowledgements}

I would like to acknowledge useful discussions and material help from many people. Adam Deller, Walter Brisken. Also many others including (in no particular order) Franco Mantovani, Steven Tingay, Walter Alef, Helge Rottman, Enno Middelberg and Richard Porcas.

\section{References}

Bhatnagar, S. 2009, Plan for Imaging Algorithm Research and Development, EVLA Memorandum 139, NRAO, [Filename evlamemo139.pdf]

Campbell, R. 2004, in European VLBI Network on New Developments in VLBI Science and Technology, ed. R. Bachiller, F. Colomer, J.-F. Desmurs, \& P. de Vicente, 245-248

Deller, A. T., Brisken, W. F., Phillips, C. J., et al. 2010, PASP, submitted

Deller, A. T., Tingay, S. J., Bailes, M., \& West, C. 2007, PASP, 119, 318

Garrett, M. A., Muxlow, T. W. B., Garrington, S. T., et al. 2001, A\&A, 366, L5

Garrett, M. A., Porcas, R. W., Pedlar, A., Muxlow, T. W. B., \& Garrington, S. T. 1999, , 43, 519

Garrett, M. A., Wrobel, J. M., \& Morganti, R. 2004, in European VLBI Network on New Developments in VLBI Science and Technology, ed. R. Bachiller, F. Colomer, J.-F. Desmurs, \& P. de Vicente, 35-38

Garrett, M. A., Wrobel, J. M., \& Morganti, R. 2005, ApJ, 619, 105

Kettenis, M., van Langevelde, H. J., Reynolds, C., \& Cotton, B. 2006, in Astronomical Society of the Pacific Conference Series, Vol. 351, Astronomical Data Analysis Software and Systems XV, ed. C. Gabriel, C. Arviset, D. Ponz, \& S. Enrique, 497-+

Lenc, E., Garrett, M. A., Wucknitz, O., Anderson, J. M., \& Tingay, S. J. 2008, ApJ, 673, 78

Middelberg, E., Deller, A., Morgan, J., et al. 2010, A\&A, accepted

Middelberg, E., Rottmann, H., Alef, W., et al. 2008, in The role of VLBI in the Golden Age for Radio Astronomy

Morgan, J. S., Mantovani, F., Deller, A. T., et al. 2010, PASP, submitted 